\title{
CONSENSUS
}

OPEN

\section{EXPERT CONSENSUS DOCUMENT}

\section{A Consensus Statement on acromegaly therapeutic outcomes}

\author{
Shlomo Melmed ${ }^{*}$, Marcello D. Bronstein ${ }^{2}$, Philippe Chanson ${ }^{3,4}$, Anne Klibanski ${ }^{5}$, \\ Felipe F. Casanueva ${ }^{6}$, John A. H. Wass ${ }^{7}$, Christian J. Strasburger ${ }^{8}$, Anton Luger ${ }^{9}$, \\ David R. Clemmons ${ }^{10}$ and Andrea Giustina ${ }^{11}$
}

\begin{abstract}
The 11th Acromegaly Consensus Conference in April 2017 was convened to update recommendations on therapeutic outcomes for patients with acromegaly. Consensus guidelines on the medical management of acromegaly were last published in 2014; since then, new pharmacological agents have been developed and new approaches to treatment sequencing have been considered. Thirty-seven experts in the management of patients with acromegaly reviewed the current literature and assessed changes in drug approvals, clinical practice standards and clinical opinion. They considered current treatment outcome goals with a focus on the impact of current and emerging somatostatin receptor ligands, growth hormone receptor antagonists and dopamine agonists on biochemical, clinical, tumour mass and surgical outcomes. The participants discussed factors that would determine pharmacological choices as well as the proposed place of each agent in the guidelines. We present consensus recommendations highlighting how acromegaly management could be optimized in clinical practice.
\end{abstract}

Acromegaly is caused by excess circulating levels of growth hormone $(\mathrm{GH})$ and insulin-like growth factor 1 (IGF1), which typically result from a GH-secreting pituitary adenoma ${ }^{1}$. Patients exhibit characteristic acral and soft tissue overgrowth (particularly in the face and hands), arthritis, jaw overbite, respiratory obstruction, hypertension and headache, as well as visual disturbances and cranial nerve palsy from tumour mass effects ${ }^{2}$. Metabolic dysfunction, including insulin resistance and elevated $\mathrm{HbA}_{1 \mathrm{c}}$, increases the risk of diabetes mellitus and cardiovascular-related morbidity and mortality ${ }^{3}$. Treatment of patients with acromegaly is aimed at normalizing GH and/or IGF1 levels to ameliorate signs and symptoms of the disease $\mathrm{e}^{2,4,5}$ and reduce excess mortality ${ }^{6-8}$.

Long-term biochemical control is achieved in fewer than $65 \%$ of patients following surgical resection of the tumour despite the use of novel surgical approaches ${ }^{9-15}$, and only approximately half of patients treated with medical therapy achieve control of IGF1 levels ${ }^{16-19}$. Radiation therapy remains an option in patients with persistently active disease, but rates of control and safety have only marginally improved with the use of stereotactic radiosurgery instead of conventional fractionated radiotherapy ${ }^{20}$. Management of acromegaly and the comorbidities of the disorder is complex and requires a comprehensive approach coordinated by a multidisciplinary team of physicians who are experts in the treatment of pituitary tumours ${ }^{21}$.
In April 2017, the Acromegaly Consensus Group convened to update the most recent consensus guidelines on the medical management of acromegaly, which were published in $2014\left(\mathrm{REF}^{4}{ }^{4}\right)$. Since that publication, new pharmacological agents have been developed and new approaches to treatment sequencing have been considered. Thirty-seven experts in acromegaly management (BOX 1) reviewed the current literature and assessed changes in drug approvals, clinical practice standards and clinical opinion since the 2014 consensus publication. Discussions focused on treatment outcome goals; effects of pharmacological agents on biochemical, clinical, tumour volume and surgical outcomes; factors determining pharmacological choices; and the proposed place of available pharmacological agents in the guidelines. Updated consensus recommendations on therapeutic outcomes for patients with acromegaly were graded using the Grading of Recommendations Assessment, Development and Evaluation system $^{22,23}$ (BOX 2), and the key recommendations are presented in BOX 3. Key changes from the 2014 consensus recommendations are presented in TABLE 1.

\section{Methods}

Meeting participants were assigned specific topics related to acromegaly treatment and outcomes. Literature searches were conducted using PubMed for Englishlanguage papers published between April 2013 and 


\section{Box 1 | 11th Acromegaly Consensus Conference participants}

Ariel Barkan (USA), Albert Beckers (Belgium), Nienke Biermasz (Netherlands), Beverly Biller (USA), Cesar Boguszewski (Brazil), Marek Bolanowski (Poland), Marcello Bronstein (Brazil), Felipe Casanueva (Spain), Philippe Chanson (France), David Clemmons (USA), Annamaria Colao (Italy), Diego Ferone (Italy), Maria Fleseriu (USA), Monica Gadelha (Brazil), Ezio Ghigo (Italy), Andrea Giustina (Italy), Mark Gurnell (UK), Anthony Heaney (USA), Andrew Hoffman (USA), Laurence Katznelson (USA), Fahrettin Kelestimur (Turkey), Anne Klibanski (USA), Steven Lamberts (Netherlands), Anton Luger (Austria), Gherardo Mazziotti (Italy), Shlomo Melmed (USA), Pietro Mortini (Italy), Marco Losa (Italy), Sebastian Neggers (Netherlands), Stephan Petersenn (Germany), Roberto Salvatori (USA), Christian Strasburger (Germany), Peter Trainer (UK), Stylianos Tsagarakis (Greece), John Wass (UK), Susan Webb (Spain) and Maria Chiara Zatelli (Italy).

March 2017. Search terms included "acromegaly" and terms associated with each topic: "biochemical outcomes", "tumour volume", "clinical symptoms", "somatostatin receptor ligand", "dopamine agonist", "GH receptor antagonist", "estrogen", "selective estrogen receptor modulator", "mortality", "complications", "surgical outcomes" and "guidelines". After a brief presentation on each topic to the entire group, participants were divided into subgroups for further discussion of the topic and reported their findings to the entire group. Participants developed consensus recommendations on the basis of all presentations, discussions and reports. All participants then voted on each recommendation. After the meeting, the Scientific Committee graded the evidence supporting the recommendations, and then graded the consensus recommendations on the basis of the quality of evidence (BOX 2).

\section{Treatment outcome goals Biochemical outcomes}

Excess GH and/or IGF1 in patients with acromegaly leads to metabolic, cardiovascular and musculoskeletal comorbidities, which, in turn, increase mortality as a result of cardiovascular, cerebrovascular and respiratory abnormalities ${ }^{1,7}$. Treatment is aimed at normalizing IGF1 levels, as doing so usually reflects adequate disease control, decreases risk of developing complications from comorbidities ${ }^{24}$ and might also reduce excess mortality ${ }^{6,25}$. However, large variability exists between

\footnotetext{
Author addresses

${ }^{1}$ Department of Medicine, Cedars-Sinai Medical Center, Los Angeles, CA, USA.

${ }^{2}$ Division of Endocrinology and Metabolism, Hospital das Clinicas, University of São Paulo, São Paulo, Brazil.

${ }^{3}$ Assistance Publique-Hôpitaux de Paris, Service d'Endocrinologie et des Maladies de la Reproduction, Centre de Référence des Maladies Rares de l'Hypophyse, Hôpital Bicêtre, Paris, France.

${ }^{4}$ UMR S-1185, Faculté de Médecine Paris-Sud, Université Paris-Sud, Université Paris-Saclay, Paris, France.

${ }^{5}$ Department of Medicine, Massachusetts General Hospital, Boston, MA, USA.

${ }^{6}$ Department of Medicine, Santiago de Compostela University, Santiago de Compostela, Spain.

${ }^{7}$ Department of Endocrinology, Churchill Hospital, Oxford, UK.

${ }^{8}$ Department of Medicine, Charité Universitätsmedizin Campus Mitte, Berlin, Germany. ${ }^{9}$ Division of Endocrinology and Metabolism, Medical University of Vienna, Vienna, Austria. ${ }^{10}$ Department of Medicine, University of North Carolina, Chapel Hill, NC, USA.

${ }^{11}$ Department of Endocrinology and Metabolism, San Raffaele University Hospital Milan, Milan, Italy.
}

the different IGF1 assays (moderate quality (MQ)). Preanalytical and analytical factors can confound results ${ }^{26}$, and differences in normative data and reference ranges make it difficult to compare results across assays ${ }^{27,28}$. It is therefore recommended that, whenever possible, endocrinologists use the same assay when monitoring IGF1 levels over time and that the selected assays adhere to accepted performance standards ${ }^{26}$ (strong recommendation (SR)). Newer techniques, such as mass spectrometry ${ }^{29}$, might offer an improvement over older immunoassays but might not be routinely available.

GH nadir levels $<1 \mu \mathrm{g} / \mathrm{l}$ after an oral glucose tolerance test (OGTT) were first defined by our Consensus Group as reflective of postoperative cure in $2000\left(\right.$ REF. $\left.^{30}\right)$. Data from large observational studies continue to show improved long-term outcomes and reduced mortality in patients who achieve $\mathrm{GH}<1 \mu \mathrm{g} / \mathrm{l}$ after surgery ${ }^{11,25,31}$ (MQ). When ultrasensitive GH assays are available, we recommend an OGTT GH cut-off of $0.4 \mu \mathrm{g} / \mathrm{l}$ (SR). Although this lower cut-off might not further improve metabolic outcomes ${ }^{32}$, nor markedly influence the percentage of patients who achieve biochemical remission ${ }^{31}$, it is better suited to the lower limits of detection of the newer assays ${ }^{33-35}$. GH nadir levels during an OGTT are also affected by factors such as patient age, BMI, sex and oestrogen use, and we recommend that these factors are considered when interpreting results of this test ${ }^{26,36}$ (discretionary recommendation (DR)).

The hypothalamic-controlled episodic pattern of GH secretion that is seen in healthy individuals is retained in patients with acromegaly ${ }^{37}$, but might not correlate with levels of IGF1 in patients who have been treated with medical therapy ${ }^{38}$ (low quality (LQ)). We recommend monitoring biochemical control by measuring both GH and IGF1 levels (SR). However, we recommend that normalizing levels of IGF1 is a key goal, as it is the best reflection of disease control ${ }^{38}$ (DR). As GH levels remain elevated with pegvisomant therapy, measuring $\mathrm{GH}$ in patients receiving pegvisomant should not be done $^{18}$ (high quality (HQ)). Monitoring of GH levels can be used to directly monitor tumour activity ${ }^{39}$ (very low quality (VLQ)), but we recommend waiting at least 12 weeks after surgery to assess IGF1 levels, as the postoperative decline in IGF1 levels can be delayed compared with that of GH levels ${ }^{11,40}$ (SR). Discordant reported IGF1 and GH values have been observed in patients following surgery as well as in those treated with somatostatin receptor ligands (SRLs) ${ }^{41,42}$ (MQ), which is probably the result of discrepancies in the assays used (MQ) and/or of biological factors, such as sex, glucose metabolism and $\mathrm{GH}$ receptor polymorphism, affecting results ${ }^{43,44}$ (VLQ). As the clinical importance of such a finding remains to be established, performing an OGTT in patients treated with an SRL is not likely to be clinically useful ${ }^{38}$.

\section{Tumour volume}

Reducing tumour size and preventing further tumour growth are clinically relevant goals for patients with acromegaly and macroadenomas $(\geq 10 \mathrm{~mm})$, as the presence of these larger tumours is independently associated with poor clinical outcomes ${ }^{45}$. Most current series evaluating tumour response to SRL therapy use a 


\section{Box 2 | Grading of evidence and recommendations}

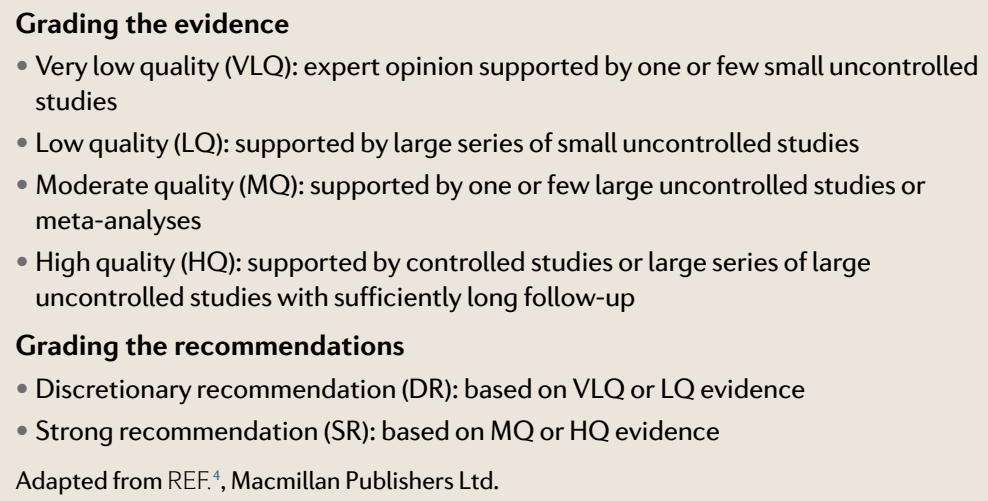

volume reduction cut-off of $20-25 \%$ to define significant reduction (LQ), as it seems unlikely that lower thresholds could be determined owing to methodological variability. However, accurately measuring volume in clinical practice might be hampered by technical differences in methods, tumour shape and intra-observer inconsistencies ${ }^{46}$ (VLQ). For routine measurements in standard clinical practice, we recommend that reduction in a single tumour dimension, such as diameter, rather than tumour volume, might be simpler to measure and is sufficient to assess meaningful mass change ${ }^{46,47}$ (DR). T2-weighted MRI hypointensity at diagnosis predicts tumour shrinkage in patients receiving SRL therapy (MQ), and we recommend that this factor might be a useful marker of tumour responsiveness ${ }^{48}(\mathrm{DR})$.

\section{Clinical symptoms}

Prevention and management of disease-associated symptoms and comorbidities are critical to improving clinical outcomes in patients with acromegaly $y^{49}$. Cardiovascular and respiratory effects are major causes of morbidity and mortality ${ }^{6,8,25}(\mathrm{HQ})$, and impaired glucose metabolism further contributes to increased cardiovascular risk ${ }^{50,51}$ (HQ). We recommend assessing and aggressively managing disease-associated comorbidities, specifically hypertension and cardiac hypertrophy, diabetes mellitus and glucose intolerance, sleep apnoea and osteopathy (SR). In patients with uncontrolled disease, these comorbidities should be aggressively managed to prevent excess mortality. When GH and/or IGF1 levels are controlled, regular 6-month follow-up is prudent. Clinician-reported outcome instruments such as SAGIT (Signs and symptoms, Associated comorbidities, GH levels, IGF1 levels and Tumour profile) and ACRODAT (Acromegaly Disease Activity Tool) provide objective measurements of acromegaly signs and symptoms, comorbidities, tumour profile, GH levels and IGF1 levels (VLQ), and we recommend that they can be used to assess and monitor indicators of disease activity ${ }^{52,53}$ (DR). Patient-reported health-related quality of life should also be considered. However, results from the acromegaly-specific questionnaire AcroQoL do not consistently correlate with biochemical control ${ }^{54-56}$, and interpretation of discordant biochemical and quality of life results remains unclear. Routine use of this tool in clinical practice is probably of limited value (DR).

\section{Pituitary tumour centres of excellence}

Treatment of acromegaly is best accomplished by a multidisciplinary team of experts meeting together in person or virtually $\mathrm{y}^{21}(\mathrm{MQ})$. With this structure, termed a pituitary tumour centre of excellence, in addition to neurosurgeons expert in transsphenoidal pituitary surgery and endocrinologists well versed in the full spectrum of medical therapies, the management team should comprise neuroradiologists well trained in pituitary and parasellar imaging; neuropathologists with expertise in molecular analysis; and radiation oncologists with specific knowledge in treating intracranial tumours (LQ). The availability of skilled nurses experienced in relevant pituitary therapies and patient education is important. We recommend that patients are treated at pituitary tumour centres of excellence to receive the best and most cost-effective care (SR). However, as patient access to such centres might be limited ${ }^{21}$, consensus recommendations are provided to optimize acromegaly therapeutic outcomes in routine clinical practice.

\section{Biochemical results of medical therapy}

Medical therapy is recommended for patients with persistent disease despite surgical resection of the adenoma as well as for patients in whom surgery is not appropriate (SR). The SRLs octreotide, lanreotide and pasireotide, as well as the dopamine agonist cabergoline, bind cognate receptors in the adenoma and suppress GH secretion; the GH antagonist pegvisomant blocks $\mathrm{GH}$ action in the periphery and blocks generation of IGF1 (REFS ${ }^{57-59}$ ).

\section{Somatostatin receptor ligands}

First-generation somatostatin receptor ligands. Biochemical control rates of approximately $55 \%$ have been reported with the first-generation SRLs octreotide and lanretotide ${ }^{60}$; however, data from rigorously conducted trials using currently available long-acting formulations show lower rates of $25-45 \% \%^{16,17,19,61}(\mathrm{MQ})$. As patient selection bias, initial IGF1 levels, previous surgery, adverse effects and treatment compliance can all impact the likelihood of achieving biochemical control, in practice, biochemical response to first-generation SRLs is likely to be higher than that observed in trials published in the past 10 years but lower than in earlier trials (LQ) ${ }^{62}$. Octreotide long-acting release (LAR) is administered once monthly by intramuscular injection; lanreotide autogel is administered once monthly subcutaneously by the patient, their caregiver or a health-care provider. As efficacy rates are similar for the two agents ${ }^{19,60}$, preference for route of delivery and/or associated cost might influence treatment choice ${ }^{63}$ (VLQ).

Studies have shown that higher doses of octreotide LAR ( $60 \mathrm{mg}$ every 28 days) as well as higher doses (180 $\mathrm{mg}$ every 28 days) and more frequent dosing ( $120 \mathrm{mg}$ every 21 days) of lanreotide autogel can improve biochemical control rates in patients who are inadequately controlled on standard doses but are responsive to SRL therapy ${ }^{64,65}(\mathrm{MQ})$. The maximal dosing of firstgeneration SRLs remains to be clarified. Careful patient selection, including considering degree of responsiveness to standard dosing, baseline IGF1 levels and 
Box 3 | Key 2018 consensus recommendations

- We recommend patients be treated at pituitary tumour centres of excellence, where possible, to receive the best and most cost-effective care.

- Surgical resection of the pituitary adenoma by an experienced neurosurgeon is recommended where possible and represents the best opportunity for cure.

- Medical therapy is recommended for patients with persistent disease despite surgical resection of the adenoma as well as patients in whom surgery is not appropriate.

- For patients with persistent disease after surgery, a first-generation long-acting somatostatin receptor ligand (SRL) is recommended as first-line therapy.

- If clinically relevant residual tumour that is unsuitable for resection is present, patients not adequately controlled on first-generation SRLs could be considered for switching to pasireotide long-acting release.

- If there is pre-existing clinically relevant impaired glucose metabolism, patients not adequately controlled on first-generation SRLs should be switched to pegvisomant.

treatment adverse effect profiles, is recommended before implementing such strategies (DR) (FIG. 1).

Second-generation somatostatin receptor ligands. Biochemical control rates with pasireotide LAR are higher than those achieved with octreotide LAR in patients who have not previously been treated with an $\mathrm{SRL}^{61}$ (MQ). However, normalized levels of IGF1 are still achieved in fewer than half of patients treated with pasireotide LAR, and nearly $70 \%$ of patients treated with pasireotide LAR exhibited hyperglycaemia-associated adverse effects $^{66}(\mathrm{MQ})$. As patients with inadequately controlled disease on octreotide LAR or lanreotide autogel show improved biochemical control after switching to pasireotide $\mathrm{LAR}^{66}$, we recommend pasireotide LAR be considered a second-line therapy (SR) (FIG. 1). Elevated $\mathrm{HbA}_{1 \mathrm{c}}$ and fasting plasma levels of glucose at baseline are strong predictors for developing hyperglycaemia during treatment with pasireotide $\mathrm{LAR}^{67}(\mathrm{MQ})$. We recommend that patients considered for treatment with pasireotide LAR should be carefully screened and monitored for glycaemic adverse effects (SR), and pasireotide LAR should preferably be used in those with normal glucose tolerance. Blood levels of glucose should be monitored weekly for the first 3 months of treatment and in the first 4-6 weeks after dose increases. Monitoring should continue throughout treatment, as clinically appropriate.

Somatostatin receptor ligands in development. New formulations of SRLs are currently in clinical development, including oral octreotide capsules, parenteral octreotide bound in a liquid crystal mix and a parenteral multi-ligand SRL with high selectivity for GH suppression ${ }^{68,69}$. A phase III study of oral octreotide in patients well controlled on octreotide LAR showed that biochemical control rates were maintained after switching to oral octreotide, and patient acceptability and compliance were improved owing to route of administration ${ }^{70}$ (LQ). Additional studies with oral octreotide are currently underway ${ }^{71,72}$.

\section{Dopamine agonist}

Cabergoline monotherapy results in biochemical control rates of approximately 35\%; similar benefits have also been seen with the addition of cabergoline to an SRL in patients with inadequate control on SRL therapy ${ }^{73}$ (LQ).
However, the benefits are largely limited to patients with mildly elevated levels of IGF1 at baseline, with the greatest benefit seen in those with IGF1 levels $\leq 1.5$ times the upper limit of normal (MQ). We recommend that cabergoline should therefore be considered as a first-line medical therapy or as an addition to first-generation SRL in patients with IGF1 levels $<2.5$ times the upper limit of normal (DR).

\section{GH receptor antagonist}

Pegvisomant monotherapy administered as secondline therapy yields biochemical control rates of $90 \%$ or more in clinical trials ${ }^{18,74}(\mathrm{HQ})$ and closer to $60 \%$ in real-world surveillance studies ${ }^{75,76}$ (MQ). This difference is probably primarily attributable to differences in doses, as patients in clinical practice are less likely to be uptitrated to the maximum dose despite higher efficacy rates being seen at higher doses ${ }^{77}$ (VLQ). Pegvisomant is approved for use at doses ranging from $10 \mathrm{mg}$ per day to $30 \mathrm{mg}$ per day, and we recommend that the daily dose should be increased to the recommended highest dose as needed (SR). Patient-specific factors such as age and BMI have been identified as predictive of the dose of pegvisomant that is required for normalization of IGF1 levels ${ }^{78,79}$ (LQ), but we recommend that physicians should regularly monitor IGF1 levels throughout therapy to determine whether normalization can be achieved by adapting the dose regimen ${ }^{59}$ (SR). Surveillance studies show that high doses of up to $60 \mathrm{mg}$ per day have been used in patients with persistently elevated IGF1 levels ${ }^{80}$; however, use of doses above $30 \mathrm{mg}$ per day is not approved, has not been prospectively studied and therefore is not recommended in clinical practice (DR).

Similarly, pegvisomant has shown high efficacy rates when given in combination with an SRL and delivered once or twice weekly ${ }^{81,82}$ (MQ) and might show continued effectiveness after discontinuing the SRL ${ }^{83}$ (LQ). Analysis of surveillance data suggests a biochemical control rate of approximately $75 \%$ in patients treated with pegvisomant monotherapy as first-line therapy ${ }^{84}$, but prospective data are lacking (VLQ).

\section{Oestrogens and SERMs}

Oestrogens and selective oestrogen receptor modulators (SERMs) reduce levels of IGF1 in patients with acromegaly when used alone or in combination with an SRL or cabergoline ${ }^{85}$ (VLQ). SERMs might have an additional benefit in men with acromegaly and hypogonadism, as these agents also increase levels of testosterone $e^{86,87}$ (VLQ). However, as published evidence is limited, optimal use of these agents remains undetermined, and sex-specific adverse effects should also be considered.

\section{Clinical outcomes of medical therapy}

Although biochemical control is the primary aim of acromegaly treatment, physicians should also consider the effect of therapy on disease-related morbidity and mortality. As a result, physicians should implement strategies to prevent, address and manage acromegaly complications. 
Table 1 | Key changes from the $\mathbf{2 0 1 4}$ to the $\mathbf{2 0 1 8}$ consensus recommendations

\begin{tabular}{|c|c|c|}
\hline Strategy & 2014 consensus recommendation ${ }^{4}$ & 2018 consensus recommendation \\
\hline $\begin{array}{l}\text { Management } \\
\text { approach }\end{array}$ & Not addressed & $\begin{array}{l}\text { Multidisciplinary team approach at a pituitary tumour } \\
\text { centre of excellence, where possible }\end{array}$ \\
\hline $\begin{array}{l}\text { Defining and } \\
\text { monitoring } \\
\text { biochemical control }\end{array}$ & $\begin{array}{l}\mathrm{GH} \text { nadir }<1 \mu \mathrm{g} / \mathrm{l} \text { after OGTT on } \\
\text { sensitive assays }\end{array}$ & $\begin{array}{l}\text { - GH nadir }<0.4 \mu \mathrm{g} / \mathrm{l} \text { after OGTT using ultrasensitive assays } \\
\text { - Wait at least } 12 \text { weeks after surgery to assess } \\
\text { IGF1 levels (delayed decline versus persistent } \\
\text { postoperative GH) } \\
\text { - Do not measure GH in patients receiving } \\
\text { pegvisomant (levels remain elevated) }\end{array}$ \\
\hline $\begin{array}{l}\text { First-line medical } \\
\text { therapy in patients } \\
\text { with persistent } \\
\text { disease after surgery }\end{array}$ & $\begin{array}{l}\text { - SRL (octreotide LAR or lanreotide } \\
\text { autogel) } \\
\text { - Cabergoline if IGF1 }<2 \text { times the } \\
\text { upper limit of normal }\end{array}$ & $\begin{array}{l}\text { - First-generation SRL (octreotide LAR or lanreotide } \\
\text { autogel) } \\
\text { - Cabergoline if IGF1 }<2.5 \text { times the upper limit of } \\
\text { normal }\end{array}$ \\
\hline $\begin{array}{l}\text { Second-line medical } \\
\text { therapy if first- } \\
\text { generation SRL is } \\
\text { not successful in } \\
\text { normalizing IGF1 }\end{array}$ & $\begin{array}{l}\text { Partial response: } \\
\text { - Increase SRL dose or decrease dose } \\
\text { interval } \\
\text { - Add pegvisomant to SRL } \\
\text { - Add cabergoline to SRL } \\
\text { Minimal or no response: } \\
\text { - Switch to pegvisomant }\end{array}$ & $\begin{array}{l}\text { Partial response: } \\
\text { - Increase first-generation SRL dose and/or increase } \\
\text { dose frequency of lanreotide autogel } \\
\text {-Add cabergoline to SRL if IGF1 is moderately elevated } \\
\text { Minimal or no response and tumour concern: } \\
\text { - Switch to pasireotide LAR } \\
\text { Minimal or no response and impaired glucose metabolism: } \\
\text { - Switch to pegvisomant } \\
\text { Minimal or no response, tumour concern and impaired } \\
\text { glucose metabolism: } \\
\text { - Add pegvisomant to first-generation SRL }\end{array}$ \\
\hline $\begin{array}{l}\text { Therapy if } \\
\text { biochemical control } \\
\text { is not achieved after } \\
\text { second-line therapy }\end{array}$ & $\begin{array}{l}\text { - Optimize pegvisomant dose } \\
\text { - Switch to pegvisomant plus } \\
\text { dopamine agonist } \\
\text { - Add dopamine agonist to SRL }\end{array}$ & $\begin{array}{l}\text { - Stereotactic radiosurgery or surgical intervention } \\
\text { (or reintervention) } \\
\text { - Temozolomide for unusually aggressive or proven } \\
\text { malignant tumours (in close cooperation with a } \\
\text { neuro-oncologist) }\end{array}$ \\
\hline $\begin{array}{l}\text { Use of clinical } \\
\text { outcome instruments }\end{array}$ & Not addressed & $\begin{array}{l}\text { - Objective tools (SAGIT and ACRODAT) can be used } \\
\text { to assess and monitor indicators of disease activity } \\
\text { - Patient quality of life questionnaires (AcroQoL) are } \\
\text { probably of limited value }\end{array}$ \\
\hline
\end{tabular}

\section{Mortality}

The increased mortality that is associated with acromegaly is largely ameliorated in patients with adequately controlled disease, who have mortality similar to that of the general population ${ }^{6,7}(\mathrm{MQ})$. In addition, patients followed up in the long term show a shift away from cardiovascular disease to cancer as a leading cause of death $^{25,88,89}$ (LQ). A continuum of benefit results from normalizing GH and IGF1 levels, leading to improved outcomes of disease-related comorbidities and reduced mortality risk ${ }^{25}$. However, the effects of specific treatment modalities on mortality in patients not cured with surgery are unclear. Data linking conventional radiotherapy with increased mortality might not apply to stereotactic radiosurgery given the potential improvements in treatment outcomes ${ }^{20,90,91}$ (VLQ), but effects on mortality have not been sufficiently investigated. Data on the long-term impact of medical therapy on all-cause mortality are few and inconclusive.

\section{Complications}

Cardiomyopathy, hypertension, valvular disease, arrhythmias and sodium and fluid retention leading to expanded extracellular fluid volume are seen in more than $60 \%$ of patients with acromegaly and are a major cause of disease-associated morbidity and mortality ${ }^{3,49}$ (HQ). Surgery, SRLs and pegvisomant can all improve left ventricular hypertrophy in patients who achieve biochemical control ${ }^{92-94}$ (MQ); improvement in hypertension and arrhythmias has also been shown in patients effectively treated with medical therapy ${ }^{93,95}$ (LQ). A study published in 2012 that examined potential adverse valvular effects associated with high-dose cabergoline in other diseases found no such effect in patients with acromegaly, which is reassuring ${ }^{96}$. As cardiac comorbidities might persist despite biochemical control of acromegaly, regular monitoring of patients is recommended (SR).

Vertebral fractures have been observed in up to $60 \%$ of patients with acromegaly ${ }^{97-99}$ (MQ). These fractures can be present despite disease control ${ }^{100,101}$ and are frequently asymptomatic ${ }^{97}$. Normal BMD on dual X-ray absorptiometry might offer false reassurance, as BMD does not predict fracture risk in patients with acromegaly ${ }^{97,98,101}(\mathrm{MQ})$. Bone turnover is probably a better indicator of bone quality ${ }^{101,102}$ (LQ), and proactive evaluations of vertebral fractures with the morphometric approach are recommended at diagnosis and annually thereafter ${ }^{103}$ (SR). Assessment of bone microarchitecture in men with acromegaly has shown that alterations in both cortical and trabecular bone occur, which further 


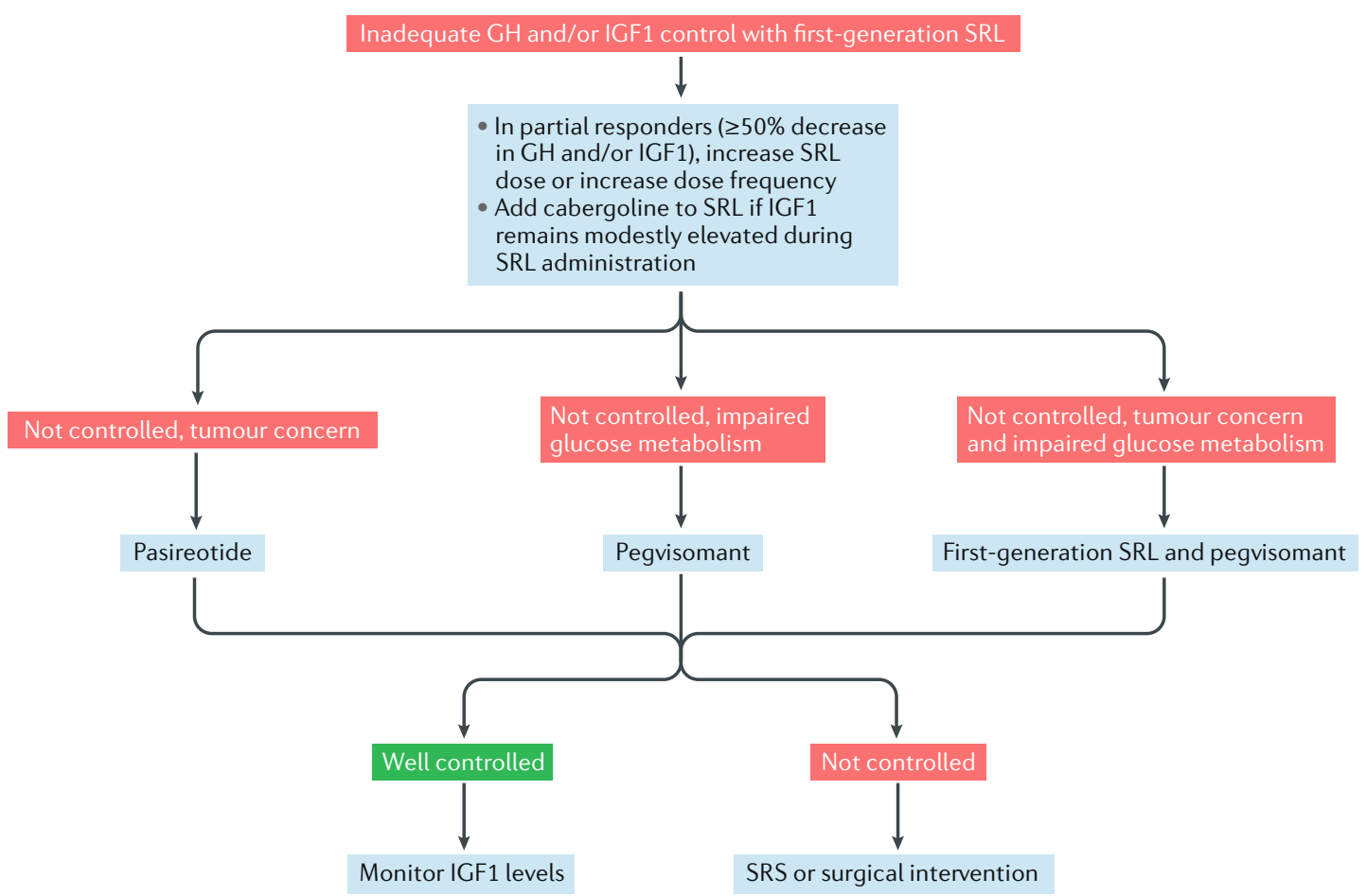

Fig. 1 A proposed algorithm for the treatment of acromegaly in patients inadequately controlled with firstgeneration somatostatin receptor ligands lanreotide autogel and octreotide long-acting release. In partial responders ( $\geq 50 \%$ decrease in growth hormone $(\mathrm{GH})$ and/or insulin-like growth factor 1 (IGF1)), increase somatostatin receptor ligand (SRL) dose and/or dose frequency. If IGF1 remains modestly elevated during SRL administration, add cabergoline to SRL. If disease control is not achieved, patients should be switched to the second-generation SRL pasireotide if there is clinically relevant residual tumour on imaging and/or clinical concern of tumour growth (tumour concern). Patients with impaired glucose tolerance should be switched to the $\mathrm{GH}$ antagonist pegvisomant. Patients with impaired glucose tolerance and tumour concern should be treated with a combination of a first-generation SRL and pegvisomant. Those who remain uncontrolled despite second-line medical therapy should be considered for stereotactic radiosurgery (SRS) or surgical intervention.

corroborates the limitations of using areal BMD to assess fracture risk in these patients ${ }^{104}$.

Soft tissue and bony craniofacial overgrowth result in considerable airway obstruction and respiratory complications in at least $25 \%$ of patients with acromegaly and might not be reversible despite the achievement of adequate biochemical control ${ }^{49}$ (MQ). We recommend that screening questionnaires for obstructive sleep apnoea are used in clinical practice, with sleep studies ordered as needed to confirm the diagnosis (SR). We also recommend that management strategies such as continuous positive airway pressure therapy should be considered for patients with persistent symptoms independent of acromegaly treatment ${ }^{105}$ (DR).

Impaired glucose metabolism and diabetes mellitus, which are present in up to half of patients with acromegaly, are infrequently affected by treatment with first-generation SRLs ${ }^{106}$ (MQ) but can be exacerbated by pasireotide ${ }^{61,67}$. By contrast, pegvisomant might have a beneficial effect on insulin sensitivity, glucose tolerance and fatty acid metabolism, mainly owing to its consequent suppression of hepatic glucose production ${ }^{107,108}$ (MQ). Close monitoring of glycaemia is recommended for all patients and particularly for those treated with pasireotide (SR). We recommend that hyperglycaemia is treated promptly (SR).
Patients with acromegaly are at increased risk of colorectal adenomatous polyps and colorectal cancer ${ }^{109}$. However, a conclusive association between the frequency of colonoscopic surveillance and cancer-specific mortality in patients with acromegaly but not concurrent highrisk factors, such as known polyps or a family history of polyps, has not been shown ${ }^{110}$ (MQ). We recommend cancer screening be carried out as recommended for the general population (DR).

\section{Tumour volume and surgical outcomes}

SRLs induce tumour shrinkage via direct and indirect antiproliferative effects ${ }^{111}$. Approximately half of patients show considerable tumour reduction within the first few months of treatment with primary or adjuvant SRLs (MQ); these changes typically, but not necessarily, correlate with biochemical control ${ }^{46,47,112-115}$ (LQ). Pasireotide might exert a greater effect on tumour control than octreotide and lanreotide ${ }^{66}$ (LQ). Patients with acromegaly owing to genetic causes, such as AIP mutations and $\mathrm{X}$-linked acrogigantism, might exhibit larger tumours that could be less responsive to therapy than tumours in patients with sporadic acromegaly ${ }^{116-118}$ (VLQ).

Although preoperative treatment with SRLs can reduce tumour size and improve surgical cure rates in patients with macroadenomas ${ }^{119,120}$ (LQ), routine use of 
SRLs for this purpose is not recommended, as evidence for a benefit on postoperative outcomes remains unclear $^{121}$ (DR).

Increased tumour growth associated with pegvisomant therapy has been reported, particularly in patients who switch from an SRL to pegvisomant ${ }^{122,123}$ (LQ). However, large observational studies carefully examining reported cases found it to be rare $^{75}$ and not more frequent than in patients on SRL therapy (MQ); furthermore, the mechanisms underlying the effect remain unclear. Nevertheless, the possibility of tumour growth with pegvisomant should be taken into consideration when selecting treatment, and ongoing imaging surveillance is advised for patients with notable residual tumour who are treated with pegvisomant (SR) (FIG. 1). Data on the effects of cabergoline on tumour volume are insufficient to form a recommendation ${ }^{58}$ (VLQ).

Imaging frequency to assess tumour volume should be individualized to each patient. We recommend that baseline tumour size and location, current medical therapy and its presumed effect on tumour mass, as well as persistent activity or biochemical relapse of the disease, should all be considered (DR).

\section{Factors in pharmacological choices}

Although the initial therapy choice will largely be driven by tumour and biochemical characteristics, we recommend that other patient-specific and disease-specific factors should be considered to appropriately individualize the therapeutic approach ${ }^{45}$ (DR). For example, although reduction of acromegaly disease activity might lead to improvements in insulin sensitivity, worsening of hyperglycaemia can occur during therapy, largely owing to inhibition of insulin secretion by SRLs (MQ). This factor is particularly relevant with the use of pasireotide but might also be relevant for the use of first-generation SRLs (LQ). Thus, for patients with impaired glucose metabolism and/or for those who experience worsening hyperglycaemia on SRL therapy, we recommend that pegvisomant or cabergoline can be considered as alternative options (DR). We also recommend that hyperglycaemia owing to acromegaly-directed therapy should be managed to aggressively control glucose levels (SR).

We recommend that tumour location (that is, proximity to the optic chiasm) as well as tumour size and the presence of local effects of the tumour mass (such as visual field defects and headache) should be used to determine treatment choice on the basis of the likely effect of therapy on tumour volume (SR).

Well-studied clinical and pathological predictors of responsiveness should also be considered. Tumours showing dense GH granulation on pathology demonstrate greater responsiveness to first-generation SRL therapy than sparsely granulated adenomas ${ }^{45,124,125}$ (LQ), whereas T2-hyperintense tumours are less likely to respond to SRL therapy than other tumours ${ }^{48,126}$ (LQ).

Other pathological markers, including immunohistochemistry to assess somatostatin receptor type 2 (SST2) and SST5 expression as well as dopamine receptor status $^{124,127}$, might be useful for individualizing treatment decisions (VLQ). These markers, however, require further prospective validation and harmonization of scoring systems to determine a personalized approach to use, as they are not approved for routine laboratory use and still remain investigational ${ }^{128}$.

\section{Proposed place in the guidelines \\ First-line medical therapy}

Surgical resection of the pituitary adenoma by an experienced neurosurgeon is recommended where possible and represents the optimal opportunity for cure (SR). Primary medical therapy with an SRL might be considered if surgery is contraindicated or if a poor likelihood of success is expected owing to patient-specific and/or tumour-specific factors (DR).

For patients with persistent disease after surgery, a first-generation long-acting SRL is recommended as first-line medical therapy (SR). The choice between octreotide LAR and lanreotide autogel is determined by availability, convenience of administration and patient preference (DR). Cabergoline can be attempted as a firstline medical therapy in patients with acromegaly and mildly elevated levels of IGF1 of $<2.5$ times the upper limit of normal (DR).

\section{Second-line medical therapy}

We recommend that additional therapies are necessary when first-line medical therapy is not successful in normalizing levels of IGF1 (SR) (FIG. 1). For patients who achieve partial response (a decrease in $\mathrm{GH}$ and/or IGF1 $250 \%$ ) after using a long-acting first-generation SRL as first-line medical therapy, we recommend that increasing the dose of the SRL and/or increasing the dose frequency of lanreotide autogel should be attempted (DR). We recommend the addition of cabergoline to continued SRL treatment when levels of IGF1 remain modestly elevated during SRL administration. If a tumoural remnant is surgically resectable, which would enable a considerable decrease in tumour mass, a second surgical intervention might be proposed before re-initiating SRL treatment.

If biochemical control is not achieved after administering the maximal dose of first-generation SRL, we recommend that treatment should be individualized on the basis of the presence or absence of clinically relevant residual tumour and impaired glucose tolerance (SR). If a clinically relevant residual tumour that is unsuitable for resection is present, we recommend that patients should be switched from first-generation SRL to pasireotide LAR (DR); if severe hyperglycaemia occurs, patients should be switched to pegvisomant (DR). However, if there is pre-existing clinically relevant impaired glucose metabolism, patients should be switched from firstgeneration SRL to pegvisomant (DR). If there is clinically relevant residual tumour and pre-existing impaired glucose metabolism, maintaining first-generation SRL and adding pegvisomant is recommended (DR).

\section{Additional considerations}

If biochemical control is not achieved after second-line therapy, stereotactic radiosurgery or surgical intervention or reintervention should be reconsidered, as appropriate (SR). Use of temozolomide should be limited to patients with unusually aggressive or proven 
malignant pituitary tumours ${ }^{129}$. In such cases, close cooperation with a neuro-oncologist is advisable (DR).

\section{Conclusions}

Our recommendations for management of acromegaly have markedly changed since the previous consensus published in 2014 (REF.). With the availability of pasireotide LAR, patients now have more treatment options and are more likely to achieve biochemical control. At the same time, clinicians should be vigilant about tailoring treatment approaches to account for the full clinical disease spectrum, taking into account biochemical control rates as well as tumour profile and glucose metabolism. Further study of current and emerging agents will help to better define the patient populations most likely to benefit from each treatment strategy and to tailor acromegaly treatments to individual patient needs.

Published online 26 July 2018
1. Melmed, S. Acromegaly pathogenesis and treatment. J. Clin. Invest. 119, 3189-3202 (2009).

2. Melmed, S. Medical progress: Acromegaly. N. Engl. J. Med. 355, 2558-2573 (2006).

3. Colao, A., Ferone, D., Marzullo, P. \& Lombardi, G. Systemic complications of acromegaly: epidemiology, pathogenesis, and management. Endocr. Rev. 25 102-152 (2004).

4. Giustina, A. et al. Expert consensus document: A consensus on the medical treatment of acromegaly. Nat. Rev. Endocrinol. 10, 243-248 (2014).

5. Katznelson, L. et al. Acromegaly: an endocrine society clinical practice guideline. J. Clin. Endocrinol. Metab. 99, 3933-3951 (2014).

6. Holdaway, I. M., Bolland, M. J. \& Gamble, G. D. A meta-analysis of the effect of lowering serum levels of GH and IGF-I on mortality in acromegaly. Eur. J. Endocrinol. 159, 89-95 (2008).

7. Dekkers, O. M., Biermasz, N. R., Pereira, A. M., Romijn, J. A. \& Vandenbroucke, J. P. Mortality in acromegaly: a metaanalysis. J. Clin. Endocrinol. Metab. 93, 61-67 (2008).

8. Sherlock, M. et al. Mortality in patients with pituitary disease. Endocr. Rev. 31, 301-342 (2010)

9. Shimon, I., Cohen, Z. R., Ram, Z. \& Hadani, M. Transsphenoidal surgery for acromegaly: endocrinological follow-up of 98 patients. Neurosurgery 48, 1239-1243; discussion 1244-1245 (2001).

10. Hazer, D. B. et al. Treatment of acromegaly by endoscopic transsphenoidal surgery: surgical experience in 214 cases and cure rates according to current consensus criteria. J. Neurosurgery 119 , 1467-1477 (2013).

11. Babu, $\mathrm{H}$. et al. Long-term endocrine outcomes following endoscopic endonasal transsphenoidal surgery for acromegaly and associated prognostic factors. Neurosurgery 81, 357-366 (2017).

12. Jane, J. A. Jr. et al. Endoscopic transsphenoidal surgery for acromegaly: remission using modern criteria, complications, and predictors of outcome. J. Clin. Endocrinol. Metab. 96, 2732-2740 (2011).

13. Anik, I. et al. Endoscopic transsphenoidal approach for acromegaly with remission rates in 401 patients: 2010 Consensus Criteria. World Neurosurg. 108 278-290 (2017)

14. Mortini, P., Barzaghi, L. R., Albano, L., Panni, P. \& Losa, M. Microsurgical therapy of pituitary adenomas. Endocrine 59, 72-81 (2018)

15. Chen, C. J. et al. Microsurgical versus endoscopic transsphenoidal resection for acromegaly: a systematic review of outcomes and complications. Acta Neurochir. 159, 2193-2207 (2017).

16. Mercado, M. et al. A prospective, multicentre study to investigate the efficacy, safety and tolerability of octreotide LAR (long-acting repeatable octreotide) in the primary therapy of patients with acromegaly. Clin. Endocrinol. 66, 859-868 (2007).

17. Melmed, S. et al. Rapid and sustained reduction of serum growth hormone and insulin-like growth factor- 1 in patients with acromegaly receiving lanreotide Autogel therapy: a randomized, placebocontrolled, multicenter study with a 52 week open extension. Pituitary 13, 18-28 (2010).

18. Trainer, P. J. et al. Treatment of acromegaly with the growth hormone-receptor antagonist pegvisomant. N. Engl. J. Med. 342, 1171-1177 (2000).

19. Murray, R. D. \& Melmed, S. A critical analysis of clinically available somatostatin analog formulations for therapy of acromegaly. J. Clin. Endocrinol. Metab. 93, 2957-2968 (2008)

20. Abu Dabrh, A. M. et al. Radiotherapy versus radiosurgery in treating patients with acromegaly: A systematic review and meta-analysis. Endocr. Pract. 21, 943-956 (2015).
21. Casanueva, F. F. et al. Criteria for the definition of Pituitary Tumor Centers of Excellence (PTCOE): A Pituitary Society Statement. Pituitary 20, 489-498 (2017).

22. Guyatt, G. H. et al. GRADE: an emerging consensus on rating quality of evidence and strength of recommendations. BMJ 336, 924-926 (2008).

23. Swiglo, B. A. et al. A case for clarity, consistency, and helpfulness: state-of-the-art clinical practice guidelines in endocrinology using the grading of recommendations, assessment, development, and evaluation system. J. Clin. Endocrinol. Metab. 93, 666-673 (2008)

24. Reid, T. J. et al. IGF-1 levels across the spectrum of normal to elevated in acromegaly: relationship to insulin sensitivity, markers of cardiovascular risk and body composition. Pituitary 18, 808-819 (2015).

25. Mercado, M. et al. Successful mortality reduction and control of comorbidities in patients with acromegaly followed at a highly specialized multidisciplinary clinic J. Clin. Endocrinol. Metab. 99, 4438-4446 (2014).

26. Clemmons, D. R. Consensus statement on the standardization and evaluation of growth hormone and insulin-like growth factor assays. Clin. Chem. 57, 555-559 (2011).

27. Chanson, P. et al. Reference values for IGF-I serum concentrations: comparison of six immunoassays. J. Clin. Endocrinol. Metab. 101, 3450-3458 (2016).

28. Mavromati, M. et al. Classification of patients with $\mathrm{GH}$ disorders may vary according to the IGF-I assay. J. Clin. Endocrinol. Metab. 102, 2844-2852 (2017).

29. Bystrom, C. et al. Clinical utility of insulin-like growth factor 1 and 2; determination by high resolution mass spectrometry. PLOS One 7, e43457 (2012).

30. Giustina, A. et al. Criteria for cure of acromegaly: a consensus statement. J. Clin. Endocrinol. Metab. 85, 526-529 (2000)

31. Starke, R. M. et al. Endoscopic versus microsurgical transsphenoidal surgery for acromegaly: outcomes in a concurrent series of patients using modern criteria for remission. J. Clin. Endocrinol. Metab. 98, 3190-3198 (2013).

32. Ku, C. R. et al. No differences in metabolic outcomes between nadir $\mathrm{GH} 0.4$ and $1.0 \mathrm{ng} / \mathrm{mL}$ during OGTT in surgically cured acromegalic patients (observational study). Medicine 95, e3808 (2016)

33. Clemmons, D. R. Clinical laboratory indices in the treatment of acromegaly. Clin. Chim. Acta 412 403-409 (2011).

34. Bidlingmaier, M. \& Freda, P. U. Measurement of human growth hormone by immunoassays: current status, unsolved problems and clinical consequences. Growth Horm. IGF Res. 20, 19-25 (2010)

35. Verrua, E. et al. Reevaluation of acromegalic patients in long-term remission according to newly proposed consensus criteria for control of disease. Int. J. Endocrinol. 2014, 581594 (2014).

36. Arafat, A. M. et al. Growth hormone response during oral glucose tolerance test: the impact of assay method on the estimation of reference values in patients with acromegaly and in healthy controls, and the role of gender, age, and body mass index. J. Clin. Endocrinol. Metab. 93, 1254-1262 (2008).

37. Ribeiro-Oliveira, A. Jr., Abrantes, M. M. \& Barkan, A. L. Complex rhythmicity and age dependence of growth hormone secretion are preserved in patients with acromegaly: further evidence for a present hypothalamic control of pituitary somatotropinomas. J. Clin. Endocrinol. Metab. 98, 2959-2966 (2013).

38. Carmichael, J. D., Bonert, V. S., Mirocha, J. M. \& Melmed, S. The utility of oral glucose tolerance testing for diagnosis and assessment of treatment outcomes in 166 patients with acromegaly. J. Clin. Endocrinol. Metab. 94, 523-527 (2009).
39. Oldfield, E. H. et al. Correlation between $\mathrm{GH}$ and IGF-1 during treatment for acromegaly. J. Neurosurgery 126, 1959-1966 (2017).

40. Shin, M. S. et al. Long-term changes in serum IGF-1 levels after successful surgical treatment of growth hormone-secreting pituitary adenoma. Neurosurgery 73, 473-479 (2013).

41. Machado, E. O. et al. Prevalence of discordant GH and IGF-I levels in acromegalics at diagnosis, after surgical treatment and during treatment with octreotide LAR. Growth Horm. IGF Res. 18, 389-393 (2008).

42. Brzana, J. A. et al. Discordant growth hormone and IGF-1 levels post pituitary surgery in patients with acromegaly naive to medical therapy and radiation: what to follow, GH or IGF-1 values? Pituitary 15 , 562-570 (2012).

43. Schilbach, K., Strasburger, C. J. \& Bidlingmaier, M. Biochemical investigations in diagnosis and follow up of acromegaly. Pituitary 20, 33-45 (2017).

44. Bianchi, A. et al. Influence of growth hormone receptor $\mathrm{d} 3$ and full-length isoforms on biochemical treatment outcomes in acromegaly. J. Clin. Endocrinol. Metab. 94, 2015-2022 (2009).

45. Cuevas-Ramos, D. et al. A structural and functional acromegaly classification. J. Clin. Endocrinol. Metab. 100, 122-131 (2015)

46. Colao, A., Auriemma, R. S. \& Pivonello, R. The effects of somatostatin analogue therapy on pituitary tumor volume in patients with acromegaly. Pituitary 19 210-221 (2016)

47. Giustina, A. et al. Meta-analysis on the effects of octreotide on tumor mass in acromegaly. PLOS One 7 , e36411 (2012).

48. Potorac, I. et al. Pituitary MRI characteristics in 297 acromegaly patients based on T2-weighted sequences. Endocr. Relat. Cancer 22, 169-177 (2015).

49. Pivonello, R. et al. Complications of acromegaly: cardiovascular, respiratory and metabolic comorbidities. Pituitary 20, 46-62 (2017).

50. Berg, C. et al. Cardiovascular risk factors in patients with uncontrolled and long-term acromegaly: comparison with matched data from the general population and the effect of disease control. J. Clin. Endocrinol. Metab. 95, 3648-3656 (2010).

51. Frara, S., Maffezzoni, F., Mazziotti, G. \& Giustina, A. Current and emerging aspects of diabetes mellitus in acromegaly. Trends Endocrinol. Metab. 27, 470-483 (2016).

52. Giustina, A. et al. SAGIT(R): clinician-reported outcome instrument for managing acromegaly in clinical practice-development and results from a pilot study. Pituitary 19, 39-49 (2016)

53. van der Lely, A. J. et al. Development of ACRODAT(R), a new software medical device to assess disease activity in patients with acromegaly. Pituitary $\mathbf{2 0}$ 692-701 (2017)

54. Webb, S. M., Badia, X., Surinach, N. L. \& Spanish AcroQol Study, G. Validity and clinical applicability of the acromegaly quality of life questionnaire, AcroOoL: a 6-month prospective study. Eur. J. Endocrinol. 155 269-277 (2006).

55. Mangupli, R., Camperos, P. \& Webb, S. M Biochemical and quality of life responses to octreotideLAR in acromegaly. Pituitary 17, 495-499 (2014).

56. Chin, S. O. et al. Change in quality of life in patients with acromegaly after treatment with octreotide LAR: first application of AcroQoL in Korea. BMJ Open $\mathbf{5}$, e006898 (2015).

57. Gadelha, M. R., Wildemberg, L. E., Bronstein, M. D., Gatto, F. \& Ferone, D. Somatostatin receptor ligands in the treatment of acromegaly. Pituitary $\mathbf{2 0}$, 100-108 (2017)

58. Kuhn, E. \& Chanson, P. Cabergoline in acromegaly. Pituitary 20, 121-128 (2017) 
59. Giustina, A. et al. Pegvisomant in acromegaly: an update. J. Endocrinol. Invest. 40, 577-589 (2017).

60. Carmichael, J. D., Bonert, V. S., Nuno, M., Ly, D. \& Melmed, S. Acromegaly clinical trial methodology impact on reported biochemical efficacy rates of somatostatin receptor ligand treatments: a metaanalysis. J. Clin. Endocrinol. Metab. 99, 1825-1833 (2014).

61. Colao, A. et al. Pasireotide versus octreotide in acromegaly: a head-to-head superiority study. J. Clin. Endocrinol. Metab. 99, 791-799 (2014).

62. Colao, A., Auriemma, R. S., Pivonello, R., Kasuki, L. \& Gadelha, M. R. Interpreting biochemical control response rates with first-generation somatostatin analogues in acromegaly. Pituitary 19, 235-247 (2016).

63. Salvatori, R. et al. Effectiveness of self- or partneradministration of an extended-release aqueous-gel formulation of lanreotide in lanreotide-naive patients with acromegaly. Pituitary 13, 115-122 (2010).

64. Giustina, A. et al. High-dose intramuscular octreotide in patients with acromegaly inadequately controlled on conventional somatostatin analogue therapy: a randomised controlled trial. Eur. J. Endocrinol. 161 331-338 (2009)

65. Giustina, A. et al. High-dose and high-frequency lanreotide autogel in acromegaly: a randomized, multicenter study. J. Clin. Endocrinol. Metab. 102, 2454-2464 (2017)

66. Gadelha, M. R. et al. Pasireotide versus continued treatment with octreotide or lanreotide in patients with inadequately controlled acromegaly (PAOLA): a randomised, phase 3 trial. Lancet Diabetes Endocrinol. 2, 875-884 (2014).

67. Schmid, H. A. et al. Effect of pasireotide on glucoseand growth hormone-related biomarkers in patients with inadequately controlled acromegaly. Endocrine 53, 210-219 (2016)

68. Melmed, S. New therapeutic agents for acromegaly. Nat. Rev. Endocrinol. 12, 90-98 (2016).

69. Maffezzoni, F., Frara, S., Doga, M., Mazziotti, G. \& Giustina, A. New medical therapies of acromegaly. Growth Horm. IGF Res. 30-31, 58-63 (2016).

70. Melmed, S. et al. Safety and efficacy of oral octreotide in acromegaly: results of a multicenter phase III trial. J. Clin. Endocrinol. Metab. 100, 1699-1708 (2015)

71. US National Library of Medicine. ClinicalTrials.gov https://clinicaltrials.gov/ct2/show/NCT03252353 (2018)

72. US National Library of Medicine. ClinicalTrials.gov https://clinicaltrials.gov/ct2/show/NCT02685709 (2018)

73. Sandret, L., Maison, P. \& Chanson, P. Place of cabergoline in acromegaly: a meta-analysis. J. Clin. Endocrinol. Metab. 96, 1327-1335 (2011).

74. van der Lely, A. J. et al. Long-term treatment of acromegaly with pegvisomant, a growth hormone receptor antagonist. Lancet 358, 1754-1759 (2001).

75. van der Lely, A. J. et al. Long-term safety of pegvisomant in patients with acromegaly: comprehensive review of 1288 subjects in ACROSTUDY. J. Clin. Endocrinol. Metab. 97, 1589-1597 (2012).

76. Freda, P. U. et al. Long-term treatment with pegvisomant as monotherapy in patients with acromegaly: Experience from acrostudy. Endocr. Pract. 21, 264-274 (2015).

77. Ragonese, M. et al. How to improve effectiveness of pegvisomant treatment in acromegalic patients. J. Endocrinol. Invest. 41, 575-581 (2017)

78. Sievers, C. et al. Prediction of therapy response in acromegalic patients under pegvisomant therapy within the German ACROSTUDY cohort. Pituitary 18, 916-923 (2015)

79. Franck, S. E. et al. A multivariable prediction model for pegvisomant dosing: monotherapy and in combination with long-acting somatostatin analogues. Eur. J. Endocrinol. 176, 421-430 (2017).

80. van der Lely, A. J. et al. Treatment with high doses of pegvisomant in 56 patients with acromegaly: experience from ACROSTUDY. Eur. J. Endocrinol. 175 239-245 (2016)

81. Neggers, S. J., de Herder, W. W., Janssen, J. A. Feelders, R. A. \& van der Lely, A. J. Combined treatment for acromegaly with long-acting somatostatin analogs and pegvisomant: long-term safety for up to 4.5 years (median 2.2 years) of follow-up in 86 patients. Eur. J. Endocrinol. 160, 529-533 (2009).

82. Neggers, S. J. et al. Long-term efficacy and safety of pegvisomant in combination with long-acting somatostatin analogs in acromegaly. J. Clin. Endocrinol. Metab. 99, 3644-3652 (2014).
83. Muhammad, A. et al. What is the efficacy of switching to weekly pegvisomant in acromegaly patients well controlled on combination therapy? Eur. J. Endocrinol. 174, 663-667 (2016).

84. Tritos, N. A. et al. Effectiveness of first-line pegvisomant monotherapy in acromegaly: an ACROSTUDY analysis. Eur. J. Endocrinol. 176, 213-220 (2017).

85. Stone, J. C., Clark, J., Cuneo, R., Russell, A. W. \& Doi, S. A. Estrogen and selective estrogen receptor modulators (SERMs) for the treatment of acromegaly: a meta-analysis of published observational studies. Pituitary 17, 284-295 (2014).

86. Balili, I. \& Barkan, A. Tamoxifen as a therapeutic agent in acromegaly. Pituitary 17, 500-504 (2014).

87. Duarte, F. H., Jallad, R. S. \& Bronstein, M. D. Clomiphene citrate for treatment of acromegaly not controlled by conventional therapies. J. Clin. Endocrinol. Metab. 100, 1863-1869 (2015).

88. Ritvonen, E. et al. Mortality in acromegaly: a 20-year follow-up study. Endocr. Relat. Cancer 23, 469-480 (2015).

89. Maione, L. et al. Changes in the management and comorbidities of acromegaly over three decades: the French Acromegaly Registry. Eur. J. Endocrinol. 176, 645-655 (2017)

90. Ayuk, J. et al. Growth hormone and pituitary radiotherapy, but not serum insulin-like growth factorconcentrations, predict excess mortality in patients with acromegaly. J. Clin. Endocrinol. Metab. 89 1613-1617 (2004).

91. Sherlock, M. et al. ACTH deficiency, higher doses of hydrocortisone replacement, and radiotherapy are independent predictors of mortality in patients with acromegaly. J. Clin. Endocrinol. Metab. 94 4216-4223 (2009).

92. Jaffrain-Rea, M. L. et al. Impact of successful transsphenoidal surgery on cardiovascular risk factors in acromegaly. Eur. J. Endocrinol. 148, 193-201 (2003).

93. Colao, A., Auriemma, R. S., Galdiero, M., Lombardi, C. $\&$ Pivonello, R. Effects of initial therapy for five years with somatostatin analogs for acromegaly on growth hormone and insulin-like growth factor-l levels, tumor shrinkage, and cardiovascular disease: a prospective study. J. Clin. Endocrinol. Metab. 94, 3746-3756 (2009).

94. Kuhn, E. et al. Long-term effects of pegvisomant on comorbidities in patients with acromegaly: a retrospective single-center study. Eur. J. Endocrinol. 173, 693-702 (2015).

95. Colao, A. et al. Efficacy of 12-month treatment with the $\mathrm{GH}$ receptor antagonist pegvisomant in patients with acromegaly resistant to long-term, high-dose somatostatin analog treatment: effect on IGF-I levels, tumor mass, hypertension and glucose tolerance. Eur. J. Endocrinol. 154, 467-477 (2006).

96. Maione, L. et al. No evidence of a detrimental effect of cabergoline therapy on cardiac valves in patients with acromegaly. J. Clin. Endocrinol. Metab. 97 E1714-1719 (2012)

97. Wassenaar, M. J. et al. High prevalence of vertebral fractures despite normal bone mineral density in patients with long-term controlled acromegaly. Eur. J. Endocrinol. 164, 475-483 (2011).

98. Claessen, K. M. et al. Progression of vertebral fractures despite long-term biochemical control of acromegaly: a prospective follow-up study. J. Clin. Endocrinol. Metab. 98, 4808-4815 (2013).

99. Bonadonna, $\mathrm{S}$. et al Increased prevalence of radiological spinal deformities in active acromegaly: a cross-sectional study in postmenopausal women. J. Bone Miner. Res. 20, 1837-1844 (2005).

100. Mazziotti, G. et al. Prevalence of vertebral fractures in men with acromegaly. J. Clin. Endocrinol. Metab. 93 4649-4655 (2008).

101. Mazziotti, G. et al. Bone turnover, bone mineral density, and fracture risk in acromegaly: a meta-analysis. J. Clin Endocrinol. Metab. 100, 384-394 (2015).

102. Parkinson, C., Kassem, M., Heickendorff, L., Flyvbjerg, A. \& Trainer, P. J. Pegvisomant-induced serum insulin-like growth factor-I normalization in patients with acromegaly returns elevated markers of bone turnover to normal. J. Clin. Endocrinol. Metab. 88, 5650-5655 (2003).

103. Mazziotti, G., Chiavistelli, S. \& Giustina, A. Pituitary diseases and bone. Endocrinol. Metab. Clin. North Am 44, 171-180 (2015)

104. Silva, P. P. B. et al. Bone microarchitecture and estimated bone strength in men with active acromegaly. Eur. J. Endocrinol. 177, 409-420 (2017).

105. Attal, P. \& Chanson, P. Endocrine aspects of obstructive sleep apnea. J. Clin. Endocrinol. Metab. 95, 483-495 (2010).
106. Mazziotti, G. et al. Effects of somatostatin analogs on glucose homeostasis: a metaanalysis of acromegaly studies. J. Clin. Endocrinol. Metab. 94, 1500-1508 (2009).

107. Drake, W. M. et al. Insulin sensitivity and glucose tolerance improve in patients with acromegaly converted from depot octreotide to pegvisomant. Eur. J. Endocrinol. 149, 521-527 (2003).

108. Higham, C. E., Rowles, S., Russell-Jones, D., Umpleby, A. M. \& Trainer, P. J. Pegvisomant improves insulin sensitivity and reduces overnight free fatty acid concentrations in patients with acromegaly. J. Clin Endocrinol. Metab. 94, 2459-2463 (2009).

109. Rokkas, T., Pistiolas, D., Sechopoulos, P., Margantinis, G $\&$ Koukoulis, G. Risk of colorectal neoplasm in patients with acromegaly: a meta-analysis. World $\mathrm{J}$. Gastroenterol. 14, 3484-3489 (2008).

110. Lois, K. et al. The role of colonoscopic screening in acromegaly revisited: review of current literature and practice guidelines. Pituitary 18, 568-574 (2015).

111. Theodoropoulou, M. \& Stalla, G. K. Somatostatin receptors: from signaling to clinical practice. Front. Neuroendocrinol. 34, 228-252 (2013).

112. Bevan, J. S. Clinical review: The antitumoral effects of somatostatin analog therapy in acromegaly. J. Clin. Endocrinol. Metab. 90, 1856-1863 (2005).

113. Melmed, S. et al. A critical analysis of pituitary tumor shrinkage during primary medical therapy in acromegaly. J. Clin. Endocrinol. Metab. 90 4405-4410 (2005).

114. Caron, P. J. et al. Tumor shrinkage with lanreotide Autogel $120 \mathrm{mg}$ as primary therapy in acromegaly: Results of a prospective multicenter clinical trial. J. Clin. Endocrinol. Metab. 99, 1282-1290 (2014).

115. Mazziotti, G. \& Giustina, A. Effects of lanreotide SR and Autogel on tumor mass in patients with acromegaly: a systematic review. Pituitary 13 60-67 (2010).

116. Daly, A. F. et al. Clinical characteristics and therapeutic responses in patients with germ-line AIP mutations and pituitary adenomas: an international collaborative study. J. Clin. Endocrinol. Metab. 95, E373-E383 (2010).

117. Beckers, A. et al. X-Linked acrogigantism syndrome clinical profile and therapeutic responses. Endocr. Relat. Cancer 22, 353-367 (2015).

118. Hernandez-Ramirez, L. C. et al. Landscape of familial isolated and young-onset pituitary adenomas: Prospective diagnosis in AIP mutation carriers. J. Clin Endocrinol. Metab. 100, E1242-E1254 (2015).

119. Carlsen, S. M. et al. Preoperative octreotide treatment in newly diagnosed acromegalic patients with macroadenomas increases cure short-term postoperative rates: a prospective, randomized trial. J. Clin. Endocrinol. Metab. 93, 2984-2990 (2008)

120. Nunes, V. S., Correa, J. M., Puga, M. E., Silva, E. M. \& Boguszewski, C. L. Preoperative somatostatin analogues versus direct transsphenoidal surgery for newly-diagnosed acromegaly patients: a systematic review and meta-analysis using the GRADE system. Pituitary 18, 500-508 (2015).

121. Fleseriu, M., Hoffman, A. R., Katznelson, L \& AACE Neuroendocrine and Pituitary Scientific Committee. American Association of Clinical Endocrinologists and American College of Endocrinology Disease State Clinical Review: management of acromegaly patients: what is the role of pre-operative medical therapy? Endocr. Pract. 21, 668-673 (2015).

122. Buhk, J. H. et al. Tumor volume of growth hormonesecreting pituitary adenomas during treatment with pegvisomant: a prospective multicenter study. J. Clin Endocrinol. Metab. 95, 552-558 (2010).

123. Marazuela, M. et al. Somatotroph tumor progression during pegvisomant therapy: a clinical and molecular study. J. Clin. Endocrinol. Metab. 96, E251-E259 (2011).

124. Brzana, J., Yedinak, C. G., Gultekin, S. H., Delashaw, J. B. \& Fleseriu, M. Growth hormone granulation pattern and somatostatin receptor subtype 2A correlate with postoperative somatostatin receptor ligand response in acromegaly: a large single center experience. Pituitary 16, 490-498 (2013).

125. Kiseljak-Vassiliades, K. et al. Growth hormone tumor histological subtypes predict response to surgical and medical therapy. Endocrine 49, 231-241 (2015).

126. Puig-Domingo, M. et al. Magnetic resonance imaging as a predictor of response to somatostatin analogs in acromegaly after surgical failure. J. Clin. Endocrinol. Metab. 95, 4973-4978 (2010).

127. Neto, L. V. et al. Expression analysis of dopamine receptor subtypes in normal human pituitaries, 
nonfunctioning pituitary adenomas and somatotropinomas, and the association between dopamine and somatostatin receptors with clinical response to octreotide-LAR in acromegaly. J. Clin Endocrinol. Metab. 94, 1931-1937 (2009).

128. Melmed, S. Pituitary medicine from discovery to patient-focused outcomes. J. Clin. Endocrinol. Metab. 101, 769-777 (2016).

129. Dillard, T. H. et al. Temozolomide for corticotroph pituitary adenomas refractory to standard therapy. Pituitary 14, 80-91 (2011).

\section{Acknowledgements}

The 11 th Acromegaly Consensus Conference was supported by educational grants from Ipsen Ltd, Novartis Pharmaceuticals and Pfizer. Scientific sponsorship of the meeting was provided by Cedars-Sinai Medical Center, Los Angeles, CA, USA.

Author contributions

All authors researched data for the article. M.D.B., P.C., A.K. F.F.C., J.A.H.W., C.J.S., A.L. and D.R.C. made substantial contributions to discussion of the content. S.M. and A.G. wrote the article. All authors reviewed and/or edited the manuscript before submission.

\section{Competing interests}

S.M. is a consultant for Chiasma, Ionis, Ipsen and Strongbridge Pharma and receives research grants from Pfizer. M.D.B. is a consultant for Ipsen and Novartis, a speakers bureau member for Ipsen and has received research grants from Novartis. P.C. has received unrestricted research and educational grants from Ipsen, Novartis and Pfizer as head of the Department of Endocrinology and Reproductive Diseases, Hôpitaux Universitaires Paris-Sud, has served as investigator for clinical trials funded by Antisense, Chiasma, Ipsen, Italpharmaco, Novartis and Pfizer and is a consultant for Ipsen, Novarti and Pfizer. All fees and honoraria are paid to his institution. A.K. is a consultant for Chiasma and Crinetics and has received research grants from Ipsen. F.F.C. is a speakers bureau member for Pfizer and has received research grants from Ipsen and Pfizer. C.J.S. is an advisory board member for Pfizer and speakers bureau member for Pfizer and Ipsen. A.L. is a consultant for Ipsen and Novartis, a speakers bureau member for Ipsen, Novartis and Pfizer and has received research grants from Pfizer. D.R.C. is a consultant for Crinetics, Ipsen and
Pfizer. A.G. is a consultant for Ipsen, Novartis and Pfizer J.A.H.W. declares no competing interests.

\section{Publisher's note}

Springer Nature remains neutral with regard to jurisdictional claims in published maps and institutional affiliations.

\section{(c) ()}

Open Access This article is licensed under a Creative Commons Attribution 4.0 International License, which permits use, sharing adaptation, distribution and reproduction in any medium or format, as long as you give appropriate credit to the original author(s) and the source, provide a link to the Creative Commons license, and indicate if changes were made. The images or other third party material in this article are included in the article's Creative Commons license, unless indicated otherwise in a credit line to the material. If material is not included in the article's Creative Commons license and your intended use is not permitted by statutory regulation or exceeds the permitted use, you will need to obtain permission directly from the copyright holder. To view a copy of this license, visit http://creativecommons.org/ licenses/by/4.0\%. 